Fisher exact test and in multivariate analysis using multiple logistic regression with bidirectional elimination based on the Akaike information criterion. In both univariate and multivariate analyses, statistical significance was corrected for multiple comparisons using the Benjamini-Hochberg method with a prespecified false discovery rate of 0.05 .

Results Complications occurred in $6.5 \%$ of procedures, including $1.9 \%$ neurological complications and $4.8 \%$ non-neurological complications. Permanent deficits occurred in only $0.2 \%$ of cases, corresponding to a single thromboembolic event. Overall, $0.5 \%$ of procedures resulted in major complications while $6.0 \%$ resulted in minor complications. Access site complications occurred in $0.7 \%$ of procedures and technical complications occurred in $0.3 \%$ of procedures. History of hypertension, history of ischemic stroke, and female sex were associated with an increased risk of complications, while femoral artery access was associated with a decreased risk of complications.

Conclusions Pediatric cerebral angiography has a low rate of major or permanent complications. Children with history of hypertension, history of ischemic stroke, or female sex are at higher risk of complications, while use of femoral access carries lower risk of complications.

Disclosures D. Lauzier: None. J. Osbun: 2; C; Medtronic, Microvention. A. Chatterjee: None. C. Moran: 2; C; Medtronic, Cerenovus. A. Kansagra: 2; C; Penumbra, Microvention, iSchemaView.

\section{E-146 THE VALUE OF LONG-TERM ANGIOGRAPHIC FOLLOW- UP FOLLOWING PIPELINE EMBOLIZATION OF INTRACRANIAL ANEURYSMS}

${ }^{1} \mathrm{D}$ Lauzier*, 'S Cler, ${ }^{2} \mathrm{~A}$ Kansagra. 'Mallinckrodt Institute of Radiology, Washington University School of Medicine, St. Louis, MO; ${ }^{2}$ Mallinckrodt Institute of Radiology, Department of Neurological Surgery, Department of Neurology, Washington University School of Medicine, St. Louis, MO

\subsection{6/neurintsurg-2021-SNIS.241}

Introduction/Purpose Flow diversion of intracranial aneurysms with the Pipeline embolization device is commonly performed, but the value of long-term angiographic follow-up has not been rigorously evaluated. Here, we examine the prevalence of actionable findings of aneurysm recurrence and development of in-stent stenosis in a cohort of patients that underwent long-term angiographic follow-up at multiple timepoints. Materials and Methods Angiographic data from eligible patients was retrospectively assessed for aneurysm occlusion, in-stent stenosis, and aneurysm regrowth or recurrence. Aneurysm occlusion was determined using the O'Kelly-Marotta scale. Patients were included in this study if they underwent angiographic imaging at 6 months post-treatment and at least one later timepoint. Pertinent findings of aneurysm occlusion status and presence of in-stent stenosis at final follow-up were stratified based on findings on 6 month angiography.

Results 100\% (132/132) of aneurysms occluded at 6 months remained occluded at final follow-up. Of those not occluded at 6 months, $85.7 \%(6 / 7)$ with an entry remnant, $56.3 \%$ (27/ $48)$ with subtotal filling, and $25 \%(6 / 24)$ with total filling at 6 months were completely occluded at final follow-up. $1.3 \%$ (2/149) of Pipeline embolization device constructs demonstrating no in-stent stenosis at 6 months developed in-stent stenosis on final angiography, while $44.4 \%$ (8/18) of PED constructs demonstrating in-stent stenosis at 6 months had

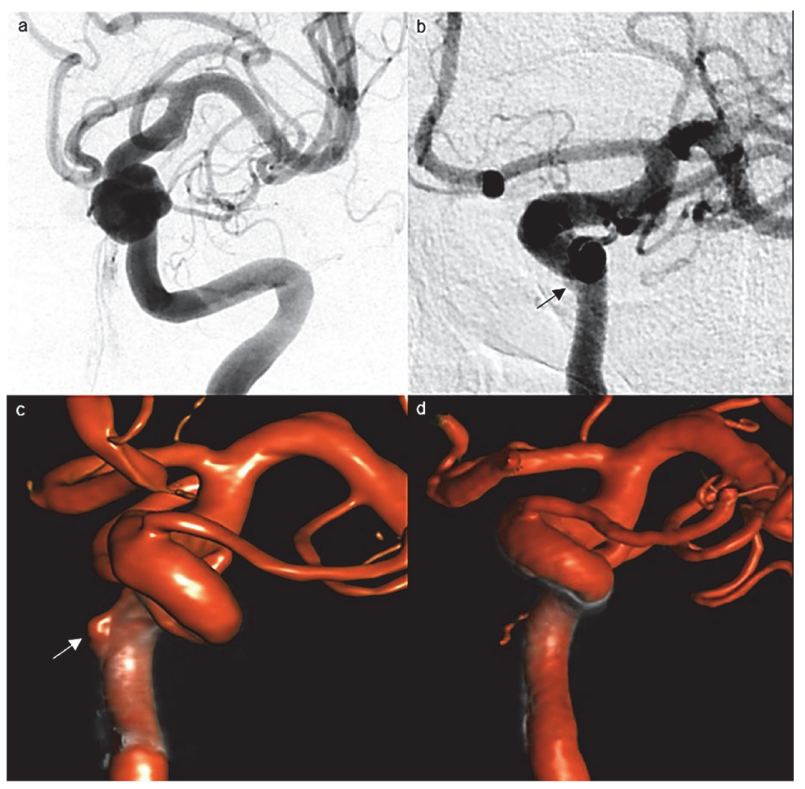

Abstract E-146 Figure 1 Preoperative angiogram showing a $9.1 \mathrm{~mm}$ saccular aneurysm at the petrous-cavernous junction of the left internal carotid artery (a), 6-month post-treatment angiography showing an entry remnant at the site of the treated aneurysm (b), 20-month posttreatment 3D rotation angiography showing a $3.4 \mathrm{~mm}$ saccular regrowth of the treated aneurysm (c), 3D rotation angiography after a second Pipeline embolization device treatment showing complete aneurysm occlusion (d).

resolution of stenosis on final angiography. No aneurysms that were completely occluded at 6 months recurred at later angiography, but one aneurysm enlarged from an entry remnant at 6 months to demonstrate subtotal filling at later follow-up (figure 1).

Conclusion Amongst patients that undergo treatment of intracranial aneurysms with Pipeline embolization device, the value of long-term angiography in patients demonstrating complete aneurysm occlusion and no in-stent stenosis on 6-month posttreatment angiography is low.

Disclosures D. Lauzier: None. S. Cler: None. A. Kansagra: 2; C; Penumbra, Microvention, iSchemaView.

\section{Late-breaking oral abstracts}

\section{LB-001 FIRST IN-HUMAN TREATMENT OF COMMUNICATING HYDROCEPHALUS USING THE CEREVASC ESHUNTTM MINIATURE BIOMIMETIC ENDOVASCULAR CSF SHUNT}

${ }^{1}$ P Lylyk*, ${ }^{1}$ I Lylyk, ${ }^{1} \mathrm{C}$ Bleise, ${ }^{1} \mathrm{~J}$ Lundquist, ${ }^{1} \mathrm{E}$ Scrivano, ${ }^{2} \mathrm{~B}$ Beneduce, ${ }^{3} \mathrm{C}$ Heilman, ${ }^{3} \mathrm{~A}$ Malek. ${ }^{1}$ Insituto Medico ENERI-Clinica La Sagrada Familia, Bs As, Argentina; ${ }^{2}$ Cerevasc Inc, Boston, MA; ${ }^{3}$ Neurosurgery, Tufts Medical Center, Boston, MA

10.1136/neurintsurg-2021-SNIS.242

Surgical ventriculoperitoneal shunting remains standard treatment for communicating hydrocephalus, despite significant infection and revision rates. A new minimally-invasive endovascular cerebrospinal fluid shunt has been developed (CereVasc eShunt ${ }^{\mathrm{TM}}$ ) to mimic arachnoid granulation function. This implant is intended to be deployed via femoral transvenous approach across the inferior petrosal sinus dura mater into the cerebellopontine angle cistern. We hereby present the first 